\title{
Volumetric reduction of cerebellar lobules associated with memory decline across the adult lifespan
}

\author{
Dong Cui ${ }^{1,2}$, Li Zhang ${ }^{2,3,4}$, Fenglian Zheng ${ }^{2,3,4}$, Huiqin Wang ${ }^{1}$, Qingjian Meng ${ }^{2}$, Wen Lu', Zhipeng Liu ${ }^{2}$, \\ Tao Yin ${ }^{1}$, Jianfeng Qiu ${ }^{2,3,4}$ \\ ${ }^{1}$ Institute of Biomedical Engineering, Chinese Academy of Medical Sciences \& Peking Union Medical College, Tianjin 300192, China; ${ }^{2}$ College \\ of Radiology, Shandong First Medical University \& Shandong Academy of Medical Sciences, Tai'an 271016, China; ${ }^{3}$ Medical Engineering and \\ Technology Research Center, ${ }^{4}$ Imaging-X Joint Laboratory, Shandong First Medical University \& Shandong Academy of Medical Sciences, Tai'an \\ 271016, China
}

Correspondence to: Tao Yin, PhD. Institute of Biomedical Engineering, Chinese Academy of Medical Sciences, Baidi Road, Tianjin 300192, China. Email: bme500@163.com; Jianfeng Qiu, PhD. Center for Medical Engineer Technology Research, Shandong First Medical University, Changcheng Road, Tai'an 271016, China. Email: jfqiu100@gmail.com.

\begin{abstract}
Background: The human cerebellum plays an essential role in motor control, is involved in cognitive function and helps to regulate emotional responses. However, little is known about the relationship between cerebellar lobules and age-related memory decline. We aimed to investigate volume alterations in cerebellar lobules at different ages and assess their correlations with reduced memory recall abilities.

Methods: A sample of 275 individuals were divided into the following four groups: 20-35 years (young), 36-50 years (early-middle age), 51-65 years (late-middle age), and 66-89 years (old). Volumes of the cerebellar lobules were obtained using volBrain software. Analysis of covariance and post hoc analysis were used to analyze group differences in cerebellar lobular volumes, and multiple comparisons were performed using the Bonferroni method. Spearman correlation was used to investigate the relationship between lobular volumes and memory recall scores.

Results: In this study, we found that older adults had smaller cerebellar volumes than the other subjects. Volumetric decreases in size were noted in bilateral lobule VI and lobule crus I. Moreover, the volumes of bilateral lobule crus I, lobule VI, and right lobule IV were significantly associated with memory recall scores.

Conclusions: In the present study, we found that some lobules of the cerebellum appear more predisposed to age-related changes than other lobules. These findings provide further evidence that specific regions of the cerebellum could be used to assess the risk of memory decline across the adult lifespan.
\end{abstract}

Keywords: Cerebellar lobules; aging; memory decline; cognitive functions

Submitted Jun 12, 2019. Accepted for publication Oct 18, 2019.

doi: $10.21037 /$ qims.2019.10.19

View this article at: http://dx.doi.org/10.21037/qims.2019.10.19

\section{Introduction}

Aging leads to a progressive deterioration in a variety of cognitive domains, including spatial processing, processing speed, working memory (WM), and long-term memory $(1,2)$. Currently, existing studies provide plenty of evidence for age-related declines in cognitive function. However, it should be noted that the existing work investigating the neural underpinnings of these declines has focused largely on the cerebral cortex (3). Mounting evidence from anatomical, clinical, and neuroimaging research indicates that the cerebellum is engaged not only in motor control, motor learning, and coordination but also in affective and higher-order cognitive processes (4-6). For instance, previous studies have shown that the lateral hemisphere of the posterior cerebellum (lobules VI-IX) and lobule X 
were associated with cognitive functions, while the anterior cerebellar lobe (lobules I-V) was primarily responsible for motor functions (7-10). Recent literature has revealed that bilateral lobule VI is associated with language paradigms, as is the right lobule VII. Therefore, the right cerebellar lobules VI and VII are believed to play a role in reading tasks (11), while the right lobule VIIIA and bilateral lobule VII are associated with working memory tasks (6). Moreover, activation of the right lobule VI and crus I of the cerebellum is associated with verbal working memory (VWM) tasks (12), while cerebellar activation during executive function has been reported in bilateral crus I (13). Furthermore, at the whole-brain level, lobule $\mathrm{X}$ is part of the default mode network (DMN) involved in attention and cognitive shifting (14).

From a neural standpoint, it has been suggested that the cerebellum plays a role in establishing the specialization of cortical regions involved in cognitive processes, and it may be crucial to the optimization of both function and structure in the developing brain $(15,16)$. Cerebellar presence in a wide range of cognitive functions has been reported in an increasing body of neuroimaging literature. A meta-analysis study demonstrated cerebellar activations in higher cognitive domains involving emotion, executive function, language, and WM (17). A large amount of neuroimaging evidence has documented that patients with cerebellar damage are impaired in WM (18-21). For example, Ravizza found that patients with cerebellar damage were moderately but consistently impaired on an immediate verbal recall task (22). A task-PET study found that cerebellar activation increased with the enhancement of working memory load (23). Previous task functional magnetic resonance imaging (fMRI) studies have also shown that different cerebellar subregions participate in processes of encoding, preservation, and retrieval output of WM $(7,24,25)$. However, notably, unlike the cerebral cortex, in which earlier damage allows for compensatory plasticity (26), cerebellar damage earlier in life can result in worse cognitive outcomes (27), leading to longterm deficits $(27,28)$.

The structural integrity of the cerebellum is a significant predictive factor of motor control and cognitive performance in an elderly population $(12,14)$. Several studies have explored the development and degeneration of regional cerebellar volume and its role in cognitive function, and they have provided key insights into the relationship between cerebellar volume and age (8-10). Bernard and Seidler found overall differences in the cerebellar volume, such that the volume was significantly smaller in older adults (8). Interestingly, however, these authors also found a significant age-by-lobule interaction, with a general pattern indicating that the anterior cerebellum and crus I were most strongly impacted by aging (8). Compared to other brain regions, the cerebellum showed significant negative correlations with advanced age, similar in magnitude to the prefrontal cortex (13). It is also worth noting that recent studies have reported important functional interactions between the cerebellum and hippocampal formation. For instance, Bernard and colleagues demonstrated differences in resting-state functional connectivity (FC) between cerebellar lobules and the hippocampus $(11,26,27)$. Therefore, the reduced cerebellar volume may contribute to memory performance deficits in older adults.

There is growing evidence for the cortical contributions to age-related declines in motor and cognitive performance; however, little is known about the relationship between cerebellar structure and memory decline across the lifespan. We hypothesized that specific lobules of the cerebellum would be associated with memory recall scores. To this end, we used a cross-sectional sample of 275 individuals, aged from 20 to 89 years, to investigate different stages of the adult lifespan with respect to alterations in cerebellar lobular volume. Furthermore, we examined the effect of age on the relationship between the cerebellar lobular gray volume and memory function.

\section{Methods}

\section{Participants}

T1-weighted image data were obtained from the Dallas Lifespan Brain Study (DLBS) database (http://fcon_1000. projects.nitrc.org/indi/retro/dlbs.html.) to form a crosssectional cohort of 275 individuals (20 to 89 years old, $54.85 \pm 20.61$ years; 174 females/101 males). Age ranges were distributed as follows: $20-35$ years (young, $n=71$ ), 3650 years (early-middle age, $n=49$ ), $51-65$ years (late-middle age, $n=54$ ) and 66-89 years (old, $n=101$ ). The participants were recruited via flyers, and media advertisements underwent health history screening via telephone and personal interviews and completed a health questionnaire. Medical history and physical examination findings were obtained for all individuals, during which their height, weight, and body mass index (BMI) were recorded. All participants were right-handed and native English speakers. Memory recall abilities were measured by the Hopkins Verbal Learning Test (28) (HVLT) (details of 
which are provided in the Supplementary I). The exclusion criteria were as follows: Mini Mental State Examination (MMSE) (29) score <26, history of neurological and psychiatric abnormalities, drug/alcohol abuse and dependence, or head injury. Each participant provided written informed consent prior to an MRI scan. The scan protocol was conducted in accordance with guidelines from the University Human Investigations Committee. All demographic and cognitive test data of subjects were collected by the DLBS database, and none of the authors was the member of the project team of the DLBS database.

\section{MRI data acquisition and analyses}

All MRI images were collected by the DLBS with a single 3.0 T Philips Achieva scanner (Amsterdam, The Netherlands) with an 8-channel head coil. The data acquired were 160 sagittal slice high-resolution T1weighted images using magnetization-prepared rapid gradient-echo (MP-RAGE) sequences with a voxel size of $1 \times 1 \times 1 \mathrm{~mm}^{3}$. The parameters used were as follows: slice thickness $=1 \mathrm{~mm}$, matrix $=256 \times 256$, field of view $(\mathrm{FOV})$ $=204 \times 256$, repetition time $(\mathrm{TR})=8.1 \mathrm{~ms}$, echo time $(\mathrm{TE})$ $=3.7 \mathrm{~ms}$, flip-angle $=12^{\circ}$.

All images were processed with the volBrain online software pipeline (http://volbrain.upv.es.). CERES2 is a new cerebellum lobule segmentation algorithm, and it became the winner of the Medical Image Computing and Computer-Assisted Intervention (MICCAI) cerebellum segmentation challenge (30). The steps of the algorithm preprocessing pipeline are outlined below. (I) DICOM images were converted to the NIfTI format. (II) The spatially adaptive non-local means filter was applied to reduce the noise in the images. (III) The N4 bias field correction was applied to correct intensity inhomogeneities across the images. This step was only applied to improve the registration process but not used as a final bias correction method. (IV) The images were linearly registered (affine transform) to the Montreal Neurological Institute (MNI) space using the MNI152 template. (V) N4 method was used to remove the inhomogeneity of the images in the MNI space. Romero et al. (31) found that the application of the N4 method in MNI space has a superior performance compared to its application on native space. (VI) The cerebellar area was cropped. (VII) The cropped area of both (the target and templates) was non-linearly registered to the cropped MNI152 atlas. (VIII) The intensity was normalized. After preprocessing, CERES2 had a library consisting of a set of cropped images and their non-linear transformations to the cropped MNI space. Finally, the cerebellum was segmented using non-local patch-based label fusion, and the Optimized PatchMatch (OPAL) algorithm was used to speed up the patch matching process (30,31). A segmentation pipeline overview can be found online (http://volbrain.upv.es/instructions.php). The cerebellar regions were segmented into 24 structures. The left and right cerebellum were delineated into lobules I-II, III, IV, V, VI, crus I, crus II, VIIB, VIIIA, VIIIB, IX, and X as shown in Figure 1.

\section{Statistical analysis}

Demographic differences among the young, early-middle age, late-middle age, and old age groups were tested using the Chi-square test for categorical variables (gender) and one-way analysis of variance (ANOVA) for continuous variables (education years, MMSE, and immediate recall and delayed recall scores) (Table 1). One-way analysis of covariance (ANCOVA) with estimated total intracranial volume (TIV) as a covariate was conducted among the four groups on cerebellar hemispheric volumes, and each of the 24 cerebellar lobular volumes. With respect to the indices with significant differences across the four groups, we examined the post hoc differences. Multiple comparisons between groups were assessed using the Bonferroni method, and statistical analysis was performed with SPSS version 22.0 for Windows (IBM Corporation, New York, USA). The level of significance was set at $\mathrm{P}<0.05$. We conducted Spearman correlation analyses between cerebellar lobular volumes with significant differences across the four groups and neurocognitive function tests, using TIV as a covariate. Bonferroni method was used to correct partial correlation results, and the level of significance was set at $\mathrm{P}<0.0019$ $(0.05 / 26)$.

\section{Results}

Demographic and cognitive functions are shown in Table 1. There were no significant group differences in gender $(\mathrm{P}=0.462)$ and years of education $(\mathrm{P}=0.338)$. However, there were significant differences among the four groups in terms of memory function $(\mathrm{P}<0.05)$. Post hoc analysis of demographics, immediate recall, and delayed recall are summarized in Table S1, which shows significant reductions in immediate recall in the old age group $(\mathrm{P}<0.05)$ compared with the other groups. Delayed recall test scores showed 

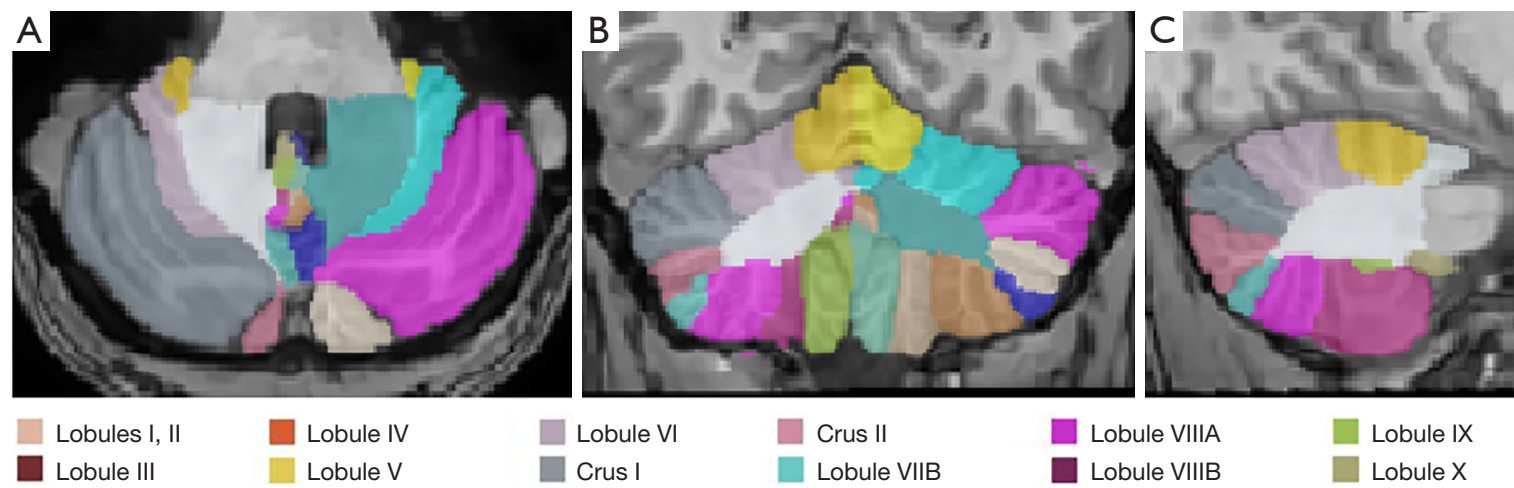

Crus I Lobule VIIB

$\square$ Lobule VIIIB

Lobule IX

Lobule X

Figure 1 Cerebellar magnetic resonance imaging (MRI) segmentation map in subjects. (A) Axial, (B) coronal and (C) sagittal; colors are given for left side labels.

Table 1 Demographic information and cognitive test scores of participants

\begin{tabular}{lcccccc}
\hline Characteristics & Young $(\mathrm{N}=71)$ & Early middle $(\mathrm{N}=49)$ & Late middle $(\mathrm{N}=54)$ & Old $(\mathrm{N}=101)$ & $\mathrm{c}^{2} / \mathrm{F}$ & $\mathrm{P}$ \\
\hline Mean age & $28.04 \pm 4.71$ & $43.61 \pm 4.67$ & $58.57 \pm 4.60$ & $77.15 \pm 6.67$ & $\mathrm{~N} . \mathrm{P}$. & $\mathrm{N} . \mathrm{P}$. \\
Gender (F/M) & $45 / 26$ & $29 / 20$ & $39 / 15$ & $61 / 40$ & $2.574^{\&}$ & 0.462 \\
Education (years) & $16.38 \pm 2.29$ & $16.07 \pm 2.16$ & $16.70 \pm 1.95$ & $16.06 \pm 2.46$ & $1.128^{\#}$ & 0.338 \\
MMSE & $28.73 \pm 1.17$ & $28.69 \pm 1.18$ & $28.50 \pm 1.09$ & $27.63 \pm 1.25$ & $16.046^{\#}$ & $<0.001^{\star *}$ \\
Immediate recall & $7.93 \pm 1.80$ & $7.35 \pm 2.02$ & $7.39 \pm 1.97$ & $6.47 \pm 1.60$ & $9.753^{\#}$ & $<0.001^{\star \star}$ \\
Delayed recall & $6.21 \pm 2.70$ & $5.47 \pm 2.98$ & $6.02 \pm 2.45$ & $4.72 \pm 2.34$ & $5.591^{\#}$ & $0.001^{\star *}$ \\
\hline
\end{tabular}

Data are expressed as mean \pm SD. N, the number of participants. MMSE, Mini-Mental State Exam; N.P., not performed. ${ }^{*} P<0.05$, ${ }^{\star \star} P<0.01$. ${ }^{\&}$ Chi-square test; "ANOVA test.

an obvious decline in the old age group $(\mathrm{P}<0.05)$ compared with the young and late-middle age groups.

\section{Group differences in cerebellar lobular volume}

We examined the differences in cerebellar lobular volumes among the four groups, as described in Table S2. There were significant group differences in the volumes of the whole bilateral cerebellum, lobule VI, lobule X, crus I and crus II, left lobules I-II, left lobule VIIIA, and left lobule VIIIB, and right lobule IV and right lobule $\mathrm{V}(\mathrm{P}<0.05)$. Pairwise comparisons of the lobular cerebellar volume are summarized in Figure 2 and Table 2. Significant differences in whole bilateral cerebellum, lobule VI, and lobule crus I, and left lobule $\mathrm{X}$ were observed in the old group compared with the other groups $(\mathrm{P}<0.05)$. Compared with the young group, the volumes of the left lobule crus II $(\mathrm{P}=0.013)$, left lobule VIIIA ( $\mathrm{P}=0.006)$, right lobule IV $(\mathrm{P}=0.013)$, right lobule V $(\mathrm{P}=0.013)$, right lobule crus II $(\mathrm{P}=0.001)$, and right lobule $\mathrm{X}(\mathrm{P}=0.009)$ showed significant alterations in the old group. In addition, left lobular I-II volume was significantly changed $(\mathrm{P}=0.019)$ in the early-middle age group relative to the old age group.

\section{Correlation between memory function and lobular volume}

Figure 3 and Table 3 show the correlation between memory function test scores and the lobular volume of the cerebellum with significant differences across the four groups. There were positive correlations between immediate recall test scores and volumes of the left lobule crus I ( $r=0.2133, \mathrm{P}=0.0004)$, right lobule IV ( $\mathrm{r}=0.2030$, $\mathrm{P}=0.0007)$, and right lobule crus $\mathrm{I}(\mathrm{r}=0.1876, \mathrm{P}=0.0018)$. Furthermore, delayed recall test scores showed positive correlations with volumes of left lobule crus I $(r=0.2153$, $\mathrm{P}=0.0003)$, left lobule VI $(\mathrm{r}=0.2499, \mathrm{P}<0.0001)$, right lobule IV ( $\mathrm{r}=0.1875, \mathrm{P}=0.0018)$, right lobule VI $(\mathrm{r}=0.2114$, $\mathrm{P}=0.0004)$, and right crus $\mathrm{I}(\mathrm{r}=0.2016, \mathrm{P}=0.0008)$. 

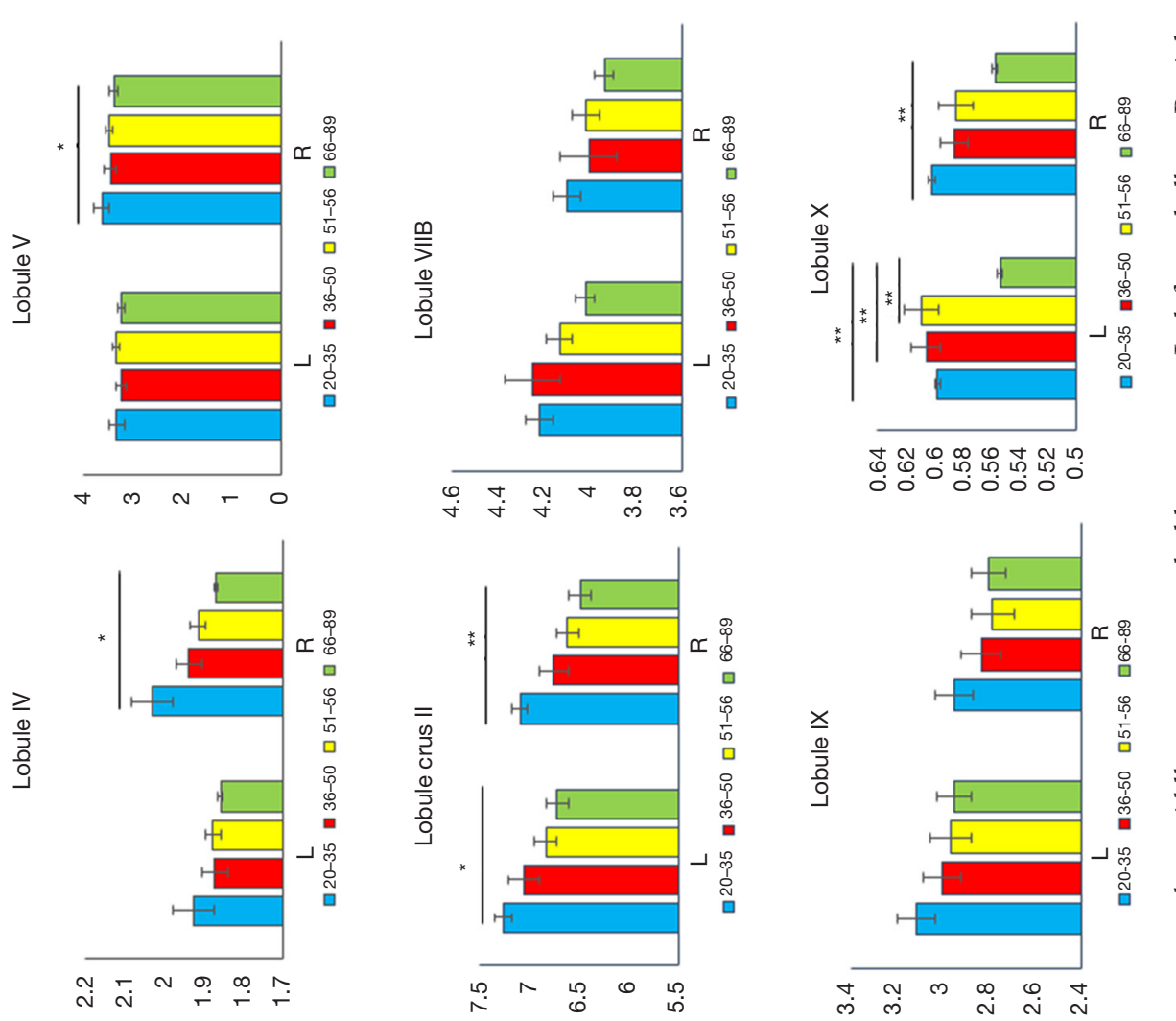

(⿸丆口
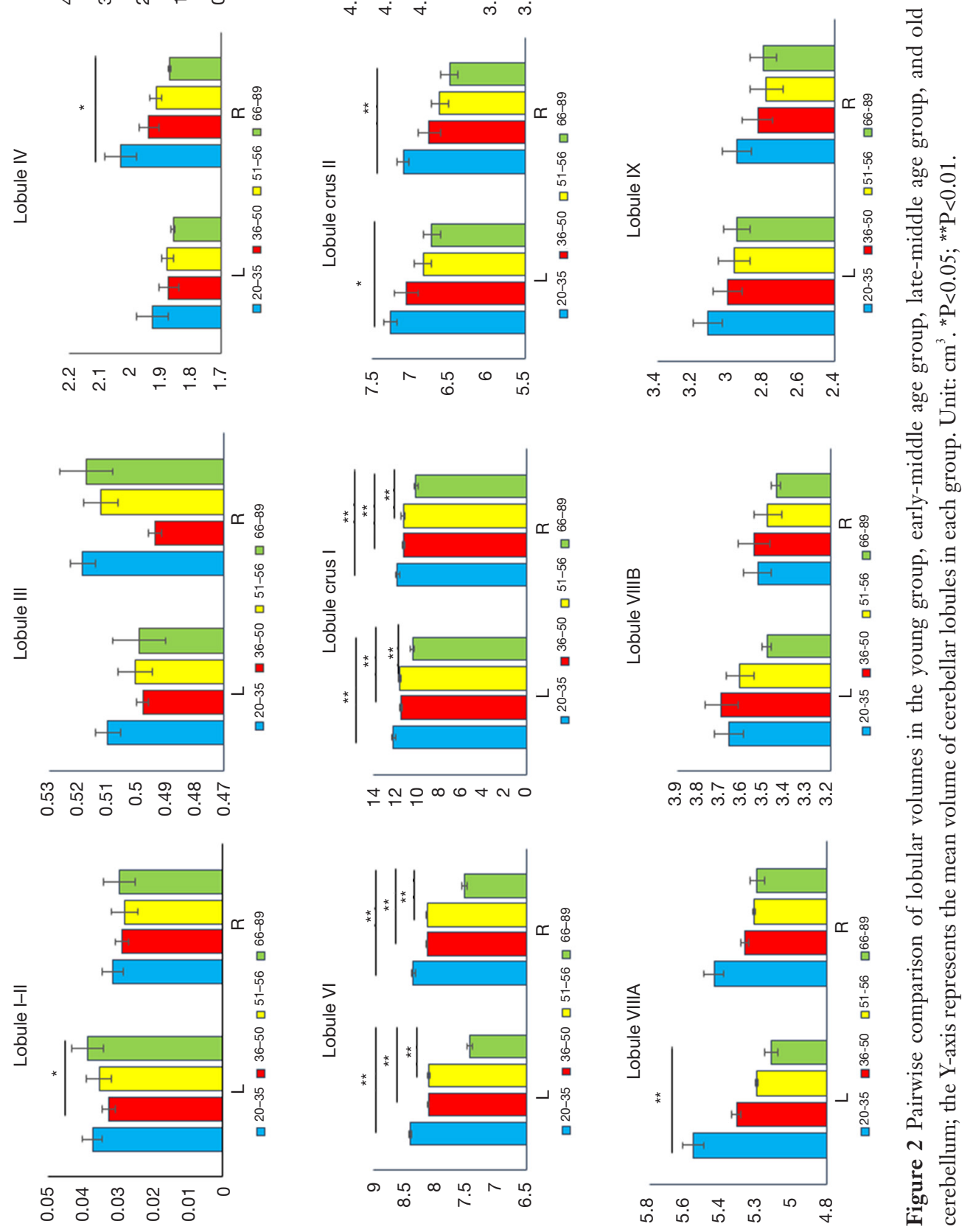
Table 2 Pairwise comparisons of lobular cerebellar volume between the young group, middle-early age group, middle-late age group, and old group

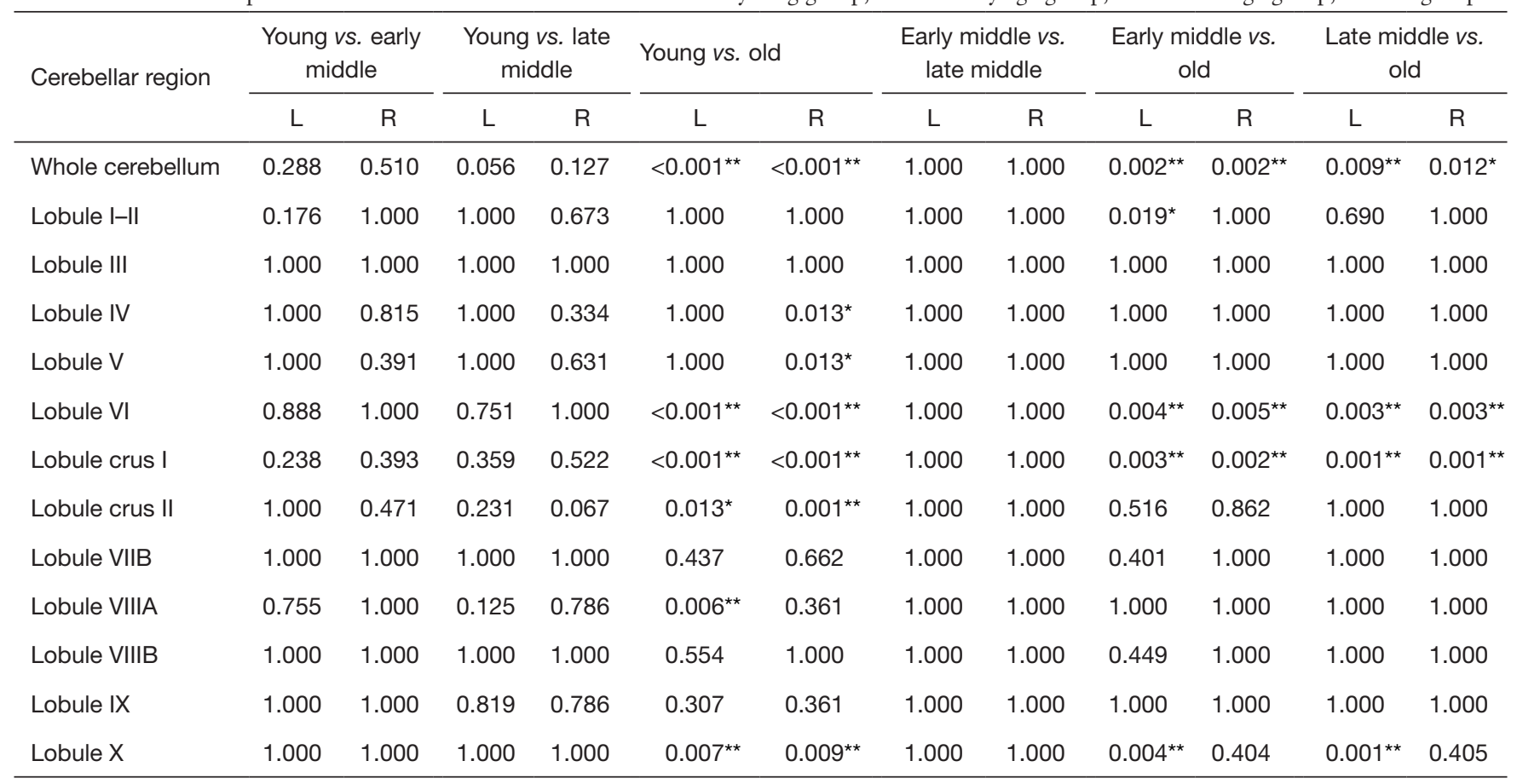

$\mathrm{L}$, left hemisphere; $\mathrm{R}$, right hemisphere. The expressed data is $\mathrm{P}$ value of the pairwise comparisons between groups, using Bonferroni method. ${ }^{*} \mathrm{P}<0.05,{ }^{* *} \mathrm{P}<0.01$.

\section{Discussion}

The present study aimed to identify volume alterations in the cerebellar lobules at different ages and assess their correlations with reduced memory recall abilities. Our findings confirmed cerebellar lobular volumetric alterations in the old group and involvement of lobule VI, lobule X, crus I, and crus II, bilaterally, and left lobule I-II, left lobule VIIIA, right lobule IV, and right lobule V. It is worth noting that the volumes of bilateral lobule VI, lobule crus I, and left lobule $\mathrm{X}$ showed significant changes in the old group compared to the other groups. Furthermore, we found that immediate recall scores were positively associated with the volumes of bilateral lobule crus I and right lobule IV. Delayed recall scores were positively associated with the volumes of bilateral lobule crus I, lobule VI, and right lobule IV. Thus, this study provides evidence for altered cerebellar lobular morphology and its association with neurocognitive deficits in aging.

\section{Age effects on cerebellar lobular volumes}

Normal aging is associated with brain structural changes, on account of extensive grey matter (GM) atrophy. The greatest shrinkage occurs in the hippocampus, caudate, prefrontal areas, and cerebellum across the lifespan (1). However, populations of neurons in the cerebellum and cerebrum are not equally affected by normal aging. The number of neurons is settled in many cerebral areas (32), whereas the anterior lobe of the cerebellum undergoes a $40 \%$ loss of both Purkinje and granule cells (33), which typically starts at the age of 50-60 years. This loss is concurrent with a volume decline in almost all cerebellar lobules (8). This difference in neuron loss between the cerebellum and cerebrum can explain why cerebellar subregions show differential losses in volume. The posterior lobe (a region more closely associated with cognitive functions) only loses about $10.6 \%$ of its volume, while the anterior lobe (which is more associated with motoric functions) loses almost $29 \%$ of its volume over the lifespan (33). MRI provides the ability to visualize the cerebellar morphology throughout the lifespan (34). A voxel-based analysis showed that an increase of mean diffusivity (MD) was observed in the cerebellar hemispheres during normal aging (35). Several MRI studies have focused on the cerebellar morphology, and they have reported decreased whole and regional 

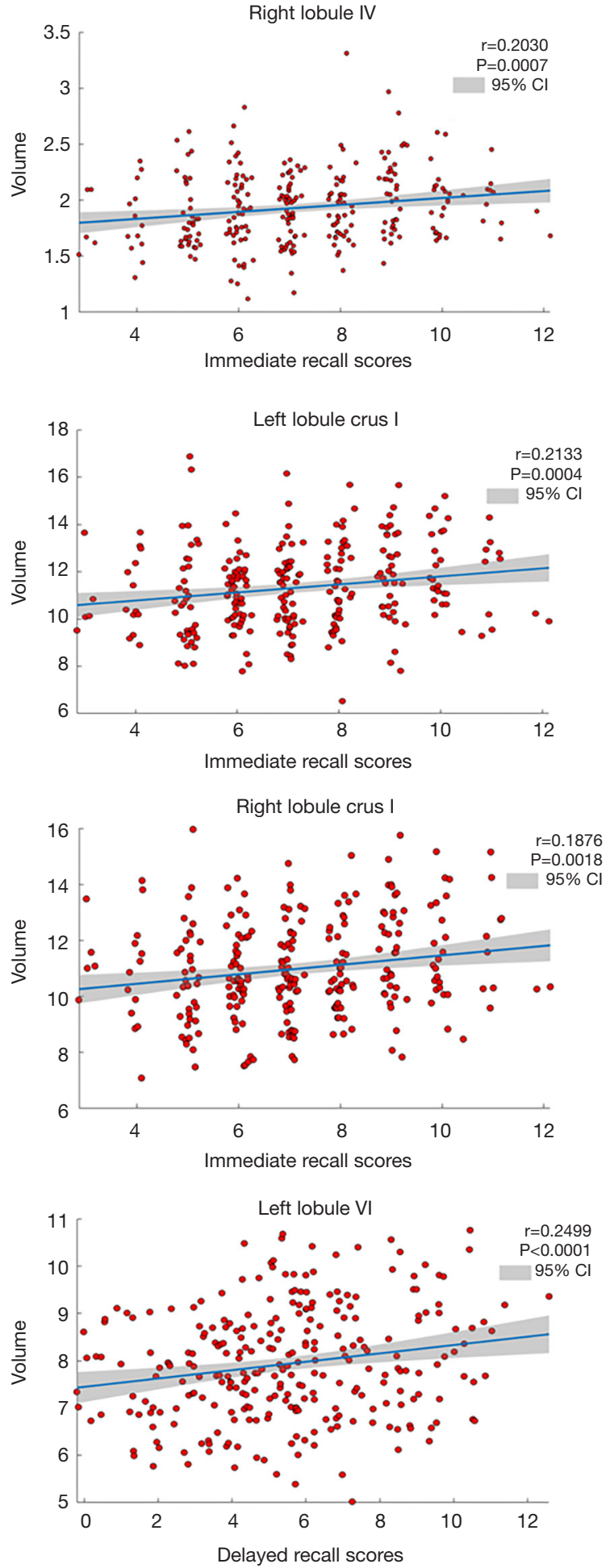
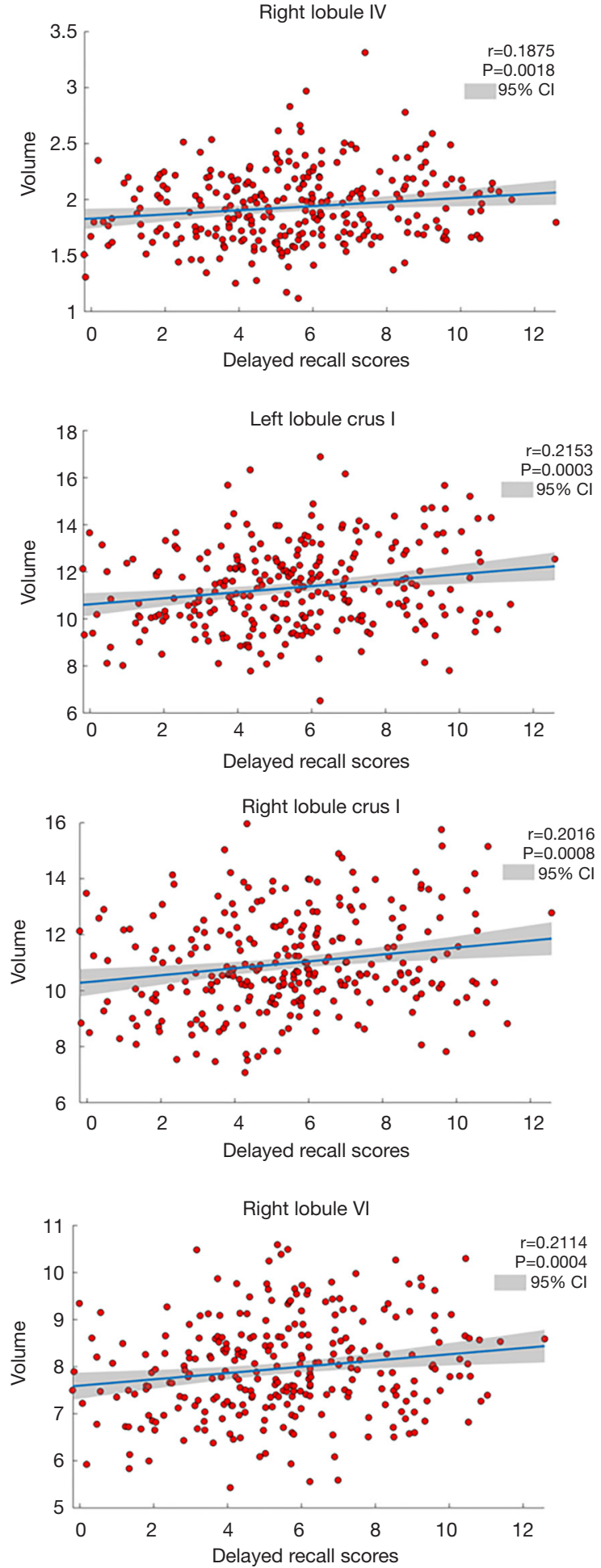

Figure 3 Correlation between lobular volumes and memory function test scores. $\mathrm{r}$ : correlation coefficient. Unit of the $\mathrm{Y}$-axis: $\mathrm{cm}^{3}$. ${ }^{*} \mathrm{P}<0.002$, ${ }^{* *} \mathrm{P}<0.0001$. 
Table 3 Correlation analysis between lobular volumes and immediate recall and delayed recall scores

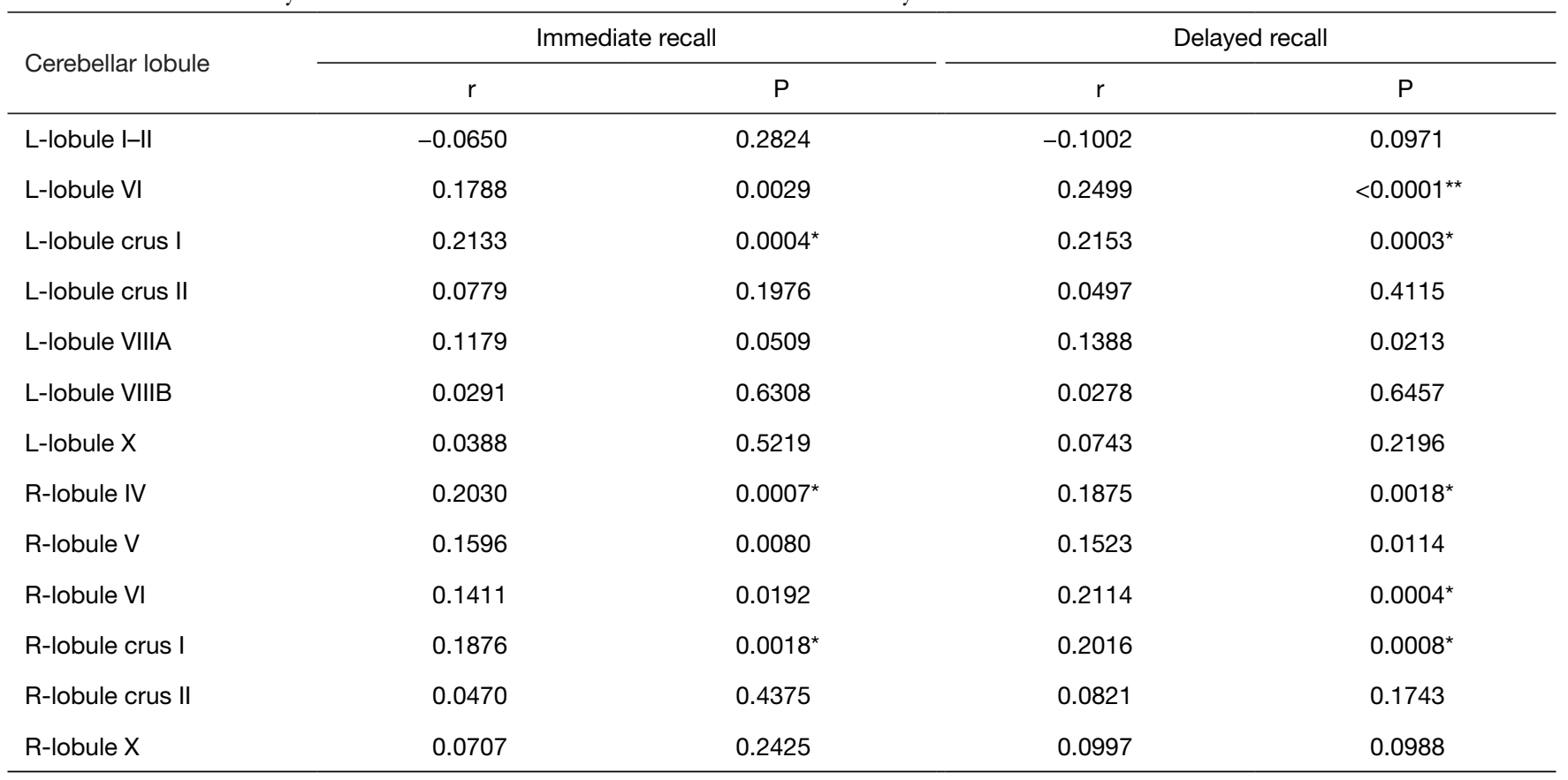

$\mathrm{L}$, left hemisphere; $\mathrm{R}$, right hemisphere. $r$, correlation coefficient. ${ }^{\star} \mathrm{P}<0.002,{ }^{\star \star} \mathrm{P}<0.0001$.

cerebellar volumes. One study found that the cerebellar lobules lose approximately $2 \%$ of their volume per decade between the ages of 20 and 80 years of age (36). Consistent with previous findings, we demonstrated that the GM of the cerebellum is reduced with aging. In the present study, the cerebellar volume was significantly smaller in the old group. Volumetric reductions in size were noted in bilateral lobule VI, lobule crus I, and crus II (37).

In the anterior lobe, we observed that there were differences in the volumes of right lobule IV and lobule $\mathrm{V}$ when compared between the young and old groups; this finding was consistent with a previous study that assessed the differences in regional cerebellar volume between the young and old age groups (8). In an earlier study, the authors found that the anterior region of the cerebellum (lobules IV and V) was correlated with visual reproduction (38). Simultaneously, a smaller volume was associated with worse performance. There were several additional relationships with lobules IV and V, including reading ability, speed of processing, and executive function (39). Previous work also has reported that the anterior/medial cerebellum (lobules IV-V) supports motor execution, including overt speech (25). Therefore, alteration of right lobule IV and right lobule $\mathrm{V}$ might influence these functions in older adults. A cross-sectional study showed that the anterior lobe and lobule VI are strong predictors of bimanual coordination performance, as they have been shown to be critical for motor tasks. Results showed that cerebellar lobule VI was a significant brain predictor of bimanual coordination performance in adolescents aged 10 to 20 years. However, in older adults aged 60 to 80 years, lobule VI, together with the primary sensorimotor cortex, formed a group of the strongest predictors. These results suggest that cerebellar lobule VI is critical for the development and preservation of bimanual coordination skills in aging (14).

A study on sensory perception has demonstrated alterations in cerebellar functional activation with advanced age. Using an odor discrimination task, Ferdon and colleagues investigated olfaction in older adults with a focus on crus I and II along with lobule VI. Older adults showed poorer performance on the odor identification test, and they also had decreased functional activity in crus I and II when compared to young adults. This study implicates lobule VI, crus I, and crus II in sensory function in older adults. Furthermore, lobule VI, lobule VIIIA, and lobule VIIIB were significantly active during auditory stimulation. Neural activity in lobules VI and VIII has been associated with accuracy on a timbre discrimination task. Patients with lesions in lobule VIIIA exhibit covert attention deficits, and lesions in the left hemispheric lobule VIIIA have 
been associated with deficits in the visual processing of biological motion, consistent with a functional role in dorsal attention network processing (40). In the current study, we demonstrated that the left cerebellar lobule VIIIA reaches a smaller volume with aging. Therefore, alteration of lobule VIIIA might influence the auditory and visual functions in older adults.

Lobule $\mathrm{X}$ is the phylogenetically oldest portion of the cerebellum, which is connected to the vestibular system and involved in balance, slower reflexive trunk movements, and related eye movements (41). Lobule $\mathrm{X}$ is part of the DMN involved in attention and cognitive shifting. Kansal et al. have provided evidence that lobule $\mathrm{X}$ dysfunction is related to impairments in executive functions (42). In nonhuman primates, lobule $\mathrm{X}$ has been found to encode components of target motion. Lobule VI and right lobule $\mathrm{X}$ are the two structures significantly active during visual stimulation (43). In studies assessing specific cerebellar regional volume deficits in patients with first-episode psychosis and schizophrenia, smaller volumes of lobule $\mathrm{X}$ have been reported (14). In this study, left lobule $X$ showed a smaller volume in the old age group compared with the other groups, and right lobule $\mathrm{X}$ showed significant alterations in the old age group relative to the young group. This result is consistent with the data reported by Hulst, who stated that the volume of lobule $\mathrm{X}$ exhibited age-related reduction (10). It is noteworthy that Hulst found a strong degeneration of lobule $\mathrm{X}$ in aging individuals, but not in cerebellar patients. In addition, we found no significant difference in the volume of hemispheric lobule IX over the adult lifespan. However, significant reductions in the GM of cerebellar hemispheric lobule IX have been reported in cases of autism spectrum disorder (ASD) and attention deficit hyperactivity disorder (ADHD), which indicates that lobule IX may become a biomarker of ASD and ADHD (44).

\section{Associations between memory and cerebellar lobules}

Aging leads to impairments in the memory function of healthy individuals. The memory dysfunction and neural mechanisms underlying these age differences in humans remain unclear. While the causes of memory decline associated with aging are multi-factorial, the integrity of the cerebellum is an important predictive factor of memory function (45). Patients with cerebellar infarcts have demonstrated memory impairments, similar to those typical for patients with frontal lesions, with WM deficits $(46,47)$. Moreover, patients with right-side lesions have been found to be impaired in verbal memory, whereas those with leftside lesions tended to be slower in a visuospatial task (46). Non-invasive cerebellar simulation studies suggest that the cerebellum contributes to storage and encoding processes involved in WM or verbal short-term memory (48). The latest mouse experiments found that optogenetic manipulation of cerebellar Purkinje cells impairs decisionmaking by reducing the ability to retain past information in WM (49) effectively. In a series of memory tests, we utilized HVLT to study age differences in memory function. The HVLT makes for an appealing measure because it provides a more detailed and standardized assessment of verbal memory than briefer tasks (28). Our results indicate that older adults manifest deficits in immediate memory recall and delayed memory recall.

The role of the cerebellum in cognitive processes is strongly supported by neuroanatomical and neuroimaging studies, which provide evidence for the existence of pathways between the prefrontal and posterior parietal cortex and the cerebellum (including lobule VI, lobule VIIA, crus I, crus II, and lobule VIIB). Previous task fMRI studies showed that lobule crus I, crus II, and lobule VIIB have been implicated in working memory and executive function, particularly in the right hemisphere $(24,25,37,50)$. During the maintenance phase of the Sternberg task, a positive linear relationship between VWM load and activity was observed in right lobule VI, extending into crus I bilaterally (51). A distinct cross-cerebro-cerebellar circuitry for VWM has been proposed with the predominant involvement of the right cerebellum, especially lobule VI (52). This could be due to anatomical connections between Broca's area, the left supplementary motor area (SMA), right lobule VI, and crus I of the cerebellum (53). It corroborates the idea that vWM is right-lateralized with a strong activation occurring mostly at the junction lobule VI/crus I. Our results showing a significantly stronger activation in the right cerebellum (lobule IV) support this hypothesis. Consistent with previous studies, we found positive correlations between immediate recall test scores and volumes of the bilateral crus I and right lobule IV. Furthermore, delayed recall test scores showed positive correlations with volumes of bilateral lobule crus I, lobule VI, and right lobule IV. Our findings confirmed that lower GM volumes of the cerebellum were associated with worse memory function, and they suggested that the volume changes in bilateral lobule VI, lobule crus I, and right lobule IV might be considered as biomarkers for aging-related memory decline.

There are several limitations to our study that should 
be considered: (I) our study was based on a cross-sectional sample, indicating that we could not investigate the changes in specific individuals over time, and future studies should examine the changes in individuals over time; (II) the age range investigated did not include the development of the cerebellum, and future studies should explore the differences before adulthood; (III) the sample size in this study was not balanced between groups; (IV) previous studies have shown that body mass index (BMI), chronic diseases, smoking, and lifestyles are risk factors for impaired memory function in older adults (54-56), and higher BMI in particular, independent of gender, and other confounding factors, is a risk factor for cognitive decline (56). All data included in this study were acquired from the DLBS dataset, so we were unable to obtain more clinical information, which may have influenced our results. We need to take these confounding factors into account in future research. Taken together, our results further highlight the importance of the cerebellum in memory function. Our data also indicate the importance of investigating the cerebellar lobular volume instead of the structure as a whole.

\section{Conclusions}

In summary, we investigated whether we could identify age differences in the cerebellar lobular volume across the adult lifespan and whether these volumes explain, in part, the differences in the memory test performance. We found that older adults had a smaller cerebellar volume than the other subjects; specifically, lobule VI and lobule crus I was more affected by age. Additionally, the volumes of bilateral lobule crus I and right lobule VI were significantly associated with memory recall scores. These findings provide further evidence that specific regions of the cerebellum could be used to assess the risk of memory decline across the adult lifespan.

\section{Acknowledgments}

The authors are very grateful to the owner of the DLBS dataset and the International Neuroimaging Data-Sharing Initiative Group for sharing this data publicly. We thank LetPub (www.letpub.com) for its linguistic assistance during the preparation of this manuscript.

Funding: We are grateful for support from the Fundamental Research Funds for the Central Universities (3332018159), the CAMS Initiative for Innovative Medicine (2016I2M-1004), and the China National Key Research and Development Program (2016YFC0103400). J. Q. was supported by the Taishan Scholars Program of Shandong Province (ts201712065).

\section{Footnote}

Conflicts of Interest: The authors have no conflicts of interest to declare.

Ethical Statement: The scan protocol was conducted in accordance with guidelines from the University Human Investigations Committee.

\section{References}

1. Park DC, Reuter-Lorenz P. The adaptive brain: aging and neurocognitive scaffolding. Annu Rev Psychol 2009;60:173.

2. Seidler RD, Bernard JA, Burutolu TB, Fling BW, Gordon MT, Gwin JT, Kwak Y, Lipps DB. Motor control and aging: Links to age-related brain structural, functional, and biochemical effects. Neuroscience \& Biobehavioral Reviews 2010;34:721-33.

3. Bernard JA, Seidler RD. Moving forward: age effects on the cerebellum underlie cognitive and motor declines. Neurosci Biobehav Rev 2014;42:193-207.

4. Hoche F, Guell X, Vangel MG, Sherman JC, Schmahmann JD. The cerebellar cognitive affective/Schmahmann syndrome scale. Brain A Journal of Neurology 2018;141:248.

5. Tedesco AM, Chiricozzi FR, Clausi S, Lupo M, Molinari M, Leggio MG. The cerebellar cognitive profile. Brain A Journal of Neurology 2011;134:3672-86.

6. Moore DM, D'Mello AM, McGrath LM, Stoodley CJ. The developmental relationship between specific cognitive domains and grey matter in the cerebellum. Dev Cogn Neurosci 2017;24:1-11.

7. Stoodley CJ, Valera EM, Schmahmann JD. An fMRI study of intra-individual functional topography in the human cerebellum. Behav Neurol 2010;23:65-79.

8. Bernard JA, Seidler RD. Relationships between regional cerebellar volume and sensorimotor and cognitive function in young and older adults. Cerebellum 2013;12:721-37.

9. Bernard JA, Leopold DR, Calhoun VD, Mittal VA. Regional cerebellar volume and cognitive function from adolescence to late middle age. Human Brain Mapping 2015;36:1102-20.

10. Hulst T, Jn VDG, Thürling M, Goericke S, Frens MA, Timmann D, Donchin O. Ageing shows a pattern of 
cerebellar degeneration analogous, but not equal, to that in patients suffering from cerebellar degenerative disease. Neuroimage 2015;116:196-206.

11. Onuki Y, Van Someren EJ, De Zeeuw CI, Van der Werf YD. Hippocampal-cerebellar interaction during spatiotemporal prediction. Cereb Cortex 2015;25:313-21.

12. Emch M, von Bastian CC, Koch K. Neural Correlates of Verbal Working Memory: An fMRI Meta-Analysis. Front Hum Neurosci 2019;13:180.

13. Jernigan TL, Archibald SL, Fennema-Notestine C, Gamst AC, Stout JC, Bonner J, Hesselink JR. Effects of age on tissues and regions of the cerebrum and cerebellum. Neurobiol Aging 2001;22:581-94.

14. Kim T, Lee KH, Oh H, Lee TY, Cho KIK, Lee J, Kwon JS. Cerebellar Structural Abnormalities Associated With Cognitive Function in Patients With First-Episode Psychosis. Front Psychiatry 2018;9:286.

15. D'Mello AM, Stoodley CJ. Cerebro-cerebellar circuits in autism spectrum disorder. Front Neurosci 2015;9:408.

16. Wang SS, Kloth AD, Badura A. The cerebellum, sensitive periods, and autism. Neuron 2014;83:518-32.

17. E KH, Chen SH, Ho MH, Desmond JE. A meta-analysis of cerebellar contributions to higher cognition from PET and fMRI studies. Hum Brain Mapp 2014;35:593-615.

18. Peterburs J, Bellebaum C, Koch B, Schwarz M, Daum I. Working memory and verbal fluency deficits following cerebellar lesions: relation to interindividual differences in patient variables. Cerebellum 2010;9:375-83.

19. Silveri MC, Di Betta AM, Filippini V, Leggio MG, Molinari M. Verbal short-term store-rehearsal system and the cerebellum. Evidence from a patient with a right cerebellar lesion. Brain 1998;121:2175-87.

20. Kirschen MP, Davis-Ratner MS, Milner MW, Chen SH, Schraedley-Desmond P, Fisher PG, Desmond JE. Verbal memory impairments in children after cerebellar tumor resection. Behav Neurol 2008;20:39-53.

21. Zhang L, Zhang L, Xue F, Yue K, Peng H, Wu Y, Sha O, Yang L, Ding Y. Brain morphological alteration and cognitive dysfunction in multiple system atrophy. Quant Imaging Med Surg 2018;8:1030-8.

22. Ravizza SM, McCormick CA, Schlerf JE, Justus T, Ivry RB, Fiez JA. Cerebellar damage produces selective deficits in verbal working memory. Brain 2006;129:306-20.

23. Schumacher EH, Lauber E, Awh E, Jonides J, Smith EE, Koeppe RA. PET evidence for an amodal verbal working memory system. Neuroimage 1996;3:79-88.

24. Stoodley CJ, Schmahmann JD. Functional topography in the human cerebellum: a meta-analysis of neuroimaging studies. Neuroimage 2009;44:489-501.

25. Marvel CL, Desmond JE. Functional topography of the cerebellum in verbal working memory. Neuropsychol Rev 2010;20:271-9.

26. Krook-Magnuson E, Armstrong C, Bui A, Lew S, Oijala M, Soltesz I. In vivo evaluation of the dentate gate theory in epilepsy. J Physiol 2015;593:2379-88.

27. Bernard JA, Peltier SJ, Wiggins JL, Jaeggi SM, Buschkuehl M, Fling BW, Kwak Y, Jonides J, Monk CS, Seidler RD. Disrupted cortico-cerebellar connectivity in older adults. Neuroimage 2013;83:103-19.

28. Grenfell-Essam R, Hogervorst E, W Rahardjo TB. The Hopkins Verbal Learning Test: an in-depth analysis of recall patterns. Memory 2018;26:385-405.

29. Cummings JL. Mini-Mental State Examination. Norms, normals, and numbers. JAMA 1993;269:2420-1.

30. Carass A, Cuzzocreo JL, Han S, Hernandez-Castillo CR, Rasser PE, Ganz M, Beliveau V, Dolz J, Ben Ayed I, Desrosiers C, Thyreau B, Romero JE, Coupe P, Manjon JV, Fonov VS, Collins DL, Ying SH, Onyike CU, Crocetti D, Landman BA, Mostofsky SH, Thompson PM, Prince JL. Comparing fully automated state-of-the-art cerebellum parcellation from magnetic resonance images. Neuroimage 2018;183:150-72.

31. Romero JE, Coupe P, Giraud R, Ta VT, Fonov V, Park MTM, Chakravarty MM, Voineskos AN, Manjon JV. CERES: A new cerebellum lobule segmentation method. Neuroimage 2017;147:916-24.

32. Peters A, Kemper T. A review of the structural alterations in the cerebral hemispheres of the aging rhesus monkey. Neurobiol Aging 2012;33:2357-72.

33. Andersen BB, Gundersen HJ, Pakkenberg B. Aging of the human cerebellum: a stereological study. J Comp Neurol 2003;466:356-65.

34. Humayun H, Yao J. Imaging the aged brain: pertinence and methods. Quant Imaging Med Surg 2019;9:842-57.

35. Abe O, Yamasue H, Aoki S, Suga M, Yamada H, Kasai K, Masutani Y, Kato N, Kato N, Ohtomo K. Aging in the CNS: comparison of gray/white matter volume and diffusion tensor data. Neurobiol Aging 2008;29:102-16.

36. Raz N, Gunning-Dixon F, Head D, Williamson A, Acker JD. Age and sex differences in the cerebellum and the ventral pons: a prospective MR study of healthy adults. AJNR Am J Neuroradiol 2001;22:1161-7.

37. Ramanoël S, Hoyau E, Kauffmann L, Renard F, Pichat C, Boudiaf N, Krainik A, Jaillard A, Baciu M. Gray Matter Volume and Cognitive Performance During Normal Aging. A Voxel-Based Morphometry Study. Front Aging 
Neurosci 2018;10:235.

38. MacLullich AM, Edmond CL, Ferguson KJ, Wardlaw JM, Starr JM, Seckl JR, Deary IJ. Size of the neocerebellar vermis is associated with cognition in healthy elderly men. Brain Cogn 2004;56:344-8.

39. Miller TD, Ferguson KJ, Reid LM, Wardlaw JM, Starr JM, Seckl JR, Deary IJ, Maclullich AM. Cerebellar vermis size and cognitive ability in community-dwelling elderly men. Cerebellum 2013;12:68-73.

40. Brissenden JA, Tobyne SM, Osher DE, Levin EJ, Halko MA, Somers DC. Topographic Cortico-cerebellar Networks Revealed by Visual Attention and Working Memory. Curr Biol 2018;28:3364-72.e5.

41. Witter L, De Zeeuw CI. In vivo differences in inputs and spiking between neurons in lobules VI/VII of neocerebellum and lobule $\mathrm{X}$ of archaeocerebellum. Cerebellum 2015;14:506-15.

42. Kansal K, Yang Z, Fishman AM, Sair HI, Ying SH, Jedynak BM, Prince JL, Onyike CU. Structural cerebellar correlates of cognitive and motor dysfunctions in cerebellar degeneration. Brain 2017;140:707-20.

43. Baumann O, Mattingley JB. Scaling of neural responses to visual and auditory motion in the human cerebellum. J Neurosci 2010;30:4489-95.

44. Stoodley CJ. Distinct regions of the cerebellum show gray matter decreases in autism, ADHD, and developmental dyslexia. Frontiers in Systems Neuroscience 2014;8:92.

45. Chiu MJ. Memory and memory disorders. Acta Neurol Taiwan 2007;16:242-50.

46. Hokkanen LS, Kauranen V, Roine RO, Salonen O, Kotila M. Subtle cognitive deficits after cerebellar infarcts. Eur J Neurol 2006;13:161-70.

47. Malm J, Kristensen B, Karlsson T, Carlberg B, Fagerlund M, Olsson T. Cognitive impairment in young adults with infratentorial infarcts. Neurology 1998;51:433-40.

Cite this article as: Cui D, Zhang L, Zheng F, Wang H, Meng Q, Lu W, Liu Z, Yin T, Qiu J. Volumetric reduction of cerebellar lobules associated with memory decline across the adult lifespan. Quant Imaging Med Surg 2020;10(1):148-159. doi: 10.21037/qims.2019.10.19
48. Pleger B, Timmann D. The role of the human cerebellum in linguistic prediction, word generation and verbal working memory: evidence from brain imaging, noninvasive cerebellar stimulation and lesion studies. Neuropsychologia 2018;115:204-10.

49. Deverett B, Kislin M, Tank DW, Wang SS. Cerebellar disruption impairs working memory during evidence accumulation. Nat Commun 2019;10:3128.

50. Stoodley CJ, Valera EM, Schmahmann JD. Functional topography of the cerebellum for motor and cognitive tasks: an fMRI study. Neuroimage 2012;59:1560-70.

51. Ashida R, Cerminara NL, Edwards RJ, Apps R, Brooks JCW. Sensorimotor, language, and working memory representation within the human cerebellum. Hum Brain Mapp 2019;40:4732-47.

52. Ng HB, Kao KL, Chan YC, Chew E, Chuang KH, Chen SH. Modality specificity in the cerebro-cerebellar neurocircuitry during working memory. Behav Brain Res 2016;305:164-73.

53. Schmahmann JD. An emerging concept. The cerebellar contribution to higher function. Arch Neurol 1991;48:1178-87.

54. McGaugh JL. Memory--a century of consolidation. Science 2000;287:248-51.

55. Chan AS, Ho YC, Cheung MC, Albert MS, Chiu HF, Lam LC. Association between mind-body and cardiovascular exercises and memory in older adults. J Am Geriatr Soc 2005;53:1754-60.

56. Gallucci M, Mazzuco S, Ongaro F, Di Giorgi E, Mecocci P, Cesari M, Albani D, Forloni GL, Durante E, Gajo GB, Zanardo A, Siculi M, Caberlotto L, Regini C. Body mass index, lifestyles, physical performance and cognitive decline: the "Treviso Longeva (TRELONG)" study. J Nutr Health Aging 2013;17:378-84. 


\section{Supplementary}

Table S1 Pairwise comparisons of demographic information and cognitive test scores between the young group, middle-early age group, middlelate age group, and old group

\begin{tabular}{|c|c|c|c|c|c|c|}
\hline Characteristics & $\begin{array}{l}\text { Young vs. } \\
\text { early-middle }\end{array}$ & $\begin{array}{l}\text { Young vs. } \\
\text { late-middle }\end{array}$ & Young vs. old & $\begin{array}{l}\text { Early-middle vs. } \\
\text { late-middle }\end{array}$ & $\begin{array}{c}\text { Early-middle vs. } \\
\text { old }\end{array}$ & $\begin{array}{c}\text { Late-middle vs. } \\
\text { old }\end{array}$ \\
\hline Gender $(F / M)^{\&}$ & 0.462 & 0.297 & 0.692 & 0.163 & 0.887 & 0.143 \\
\hline Immediate recall ${ }^{\#}$ & 0.499 & 0.588 & $<0.001^{* *}$ & 1.000 & $0.032^{*}$ & $0.016^{*}$ \\
\hline Delayed recall ${ }^{\#}$ & 0.735 & 1.000 & $0.001^{*}$ & 1.000 & 0.584 & $0.019^{*}$ \\
\hline
\end{tabular}

\&, Chi-square test; ", the expressed data is $\mathrm{P}$ value of the pairwise comparisons between groups, using Bonferroni method. "P<0.05, ${ }^{*} \mathrm{P}<0.01$. MMSE, Mini-Mental State Exam.

Table S2 Statistical analysis of lobular volumes among the 4 groups

\begin{tabular}{|c|c|c|c|c|c|c|c|c|}
\hline \multirow{2}{*}{ Cerebellar region } & \multicolumn{4}{|c|}{ L } & \multicolumn{4}{|c|}{$\mathrm{R}$} \\
\hline & $\mathrm{F}^{\mathrm{a}}$ & $\mathrm{P}^{\mathrm{a}}$ & $F^{b}$ & $\mathrm{P}^{\mathrm{b}}$ & $\mathrm{F}^{\mathrm{a}}$ & $P^{a}$ & $F^{b}$ & $P^{b}$ \\
\hline Whole cerebellum & 14.926 & $<0.001^{\star *}$ & 33.176 & $<0.001^{\star \star}$ & 13.230 & $<0.001^{\star *}$ & 28.242 & $<0.001^{\star *}$ \\
\hline Lobule I-II & 3.212 & $0.023^{*}$ & 2.859 & $0.037^{\star}$ & 0.959 & 0.413 & 0.904 & 0.440 \\
\hline Lobule III & 0.166 & 0.919 & 0.311 & 0.817 & 0.514 & 0.673 & 0.418 & 0.740 \\
\hline Lobule V & 0.804 & 0.492 & 1.517 & 0.210 & 3.257 & $0.022^{*}$ & 4.430 & $0.005^{\star \star}$ \\
\hline Lobule VI & 11.730 & $<0.001^{\star *}$ & 15.633 & $<0.001^{\star *}$ & 10.301 & $<0.001^{* *}$ & 14.562 & $<0.001^{\star \star}$ \\
\hline Lobule crus I & 14.826 & $<0.001^{\star *}$ & 21.947 & $<0.001^{\star \star}$ & 14.548 & $<0.001^{\star *}$ & 21.328 & $<0.001^{\star *}$ \\
\hline Lobule crus II & 3.515 & $0.016^{\star \star}$ & 4.609 & $0.004^{\star \star}$ & 4.946 & $0.002^{\star \star}$ & 6.226 & $<0.001^{\star *}$ \\
\hline Lobule VIIIB & 1.491 & 0.217 & 3.725 & $0.012^{*}$ & 0.371 & 0.774 & 1.290 & 0.278 \\
\hline Lobule IX & 1.394 & 0.245 & 2.106 & 0.100 & 1.426 & 0.235 & 1.903 & 0.129 \\
\hline Lobule X & 7.172 & $<0.001^{\star *}$ & 8.636 & $<0.001^{\star *}$ & 3.690 & $0.012^{*}$ & 4.498 & $0.004^{\star *}$ \\
\hline
\end{tabular}

$\mathrm{L}$, left hemisphere; R, right hemisphere; ${ }^{\star} \mathrm{P}<0.05$, ${ }^{\star \star} \mathrm{P}<0.01 .{ }^{a}$, ANOVA test, no covariates; ${ }^{\mathrm{b}}$, ANCOVA test, controlling for estimated total intracranial volume. 


\section{Supplementary I Hopkins verbal learning task (HVLT)}

The HVLT consists of a 12-item word list, comprising four words from each of the three well-known semantic categories (Table S3). A total recall score and a learning index are calculated. After 20 minutes, delayed recall of the word list is tested.

\section{Task 1}

Immediate recall: Participants listen to a list of 12 words and are then asked to recall as many words from the list as they can.

Say: "In this task, I will read a list of words aloud. Please try to remember as many words as you can. After I finish, I want you to repeat as many of the words you can recall." Then read each word clearly at a rate of about 1.5 seconds per word.

Say: "Ok. Now tell me as many words as you can remember from that list."

\section{Task 2 (20 min after Task 1)}

Delay recall: Approximately 20 minutes after the initial word learning, participants are asked again to recall as many words as they can remember from the list.

Say: "A while ago, I read you a list of words and asked you to recall as many of the words as possible. Now I want you to recall the words I read for you from the list. Please just try to remember as many words as you can."

\section{Outcome measures}

Immediate recall: the number of words correctly recalled.

Delayed recall: the number of words correctly recalled after a 20-minute delay.

Table S3 The word list of the Hopkins verbal learning task (HVLT)

\begin{tabular}{l} 
Word Immediate \\
\hline Teacher \\
Basketball \\
Lettuce \\
Dentist \\
Tennis \\
Bean \\
Engineer \\
Potato \\
Professor \\
Golf \\
Corn \\
Soccer \\
\# correct
\end{tabular}

